\title{
Ultrasound-modulated optical microscopy for ex-vivo imaging of scattering biological tissue
}

Sri-Rajasekhar Kothapalli, Lihong V. Wang

Sri-Rajasekhar Kothapalli, Lihong V. Wang, "Ultrasound-modulated optical microscopy for ex-vivo imaging of scattering biological tissue," Proc. SPIE 7177, Photons Plus Ultrasound: Imaging and Sensing 2009, 71772N (24 February 2009); doi: 10.1117/12.810566

SPIE. Event: SPIE BiOS, 2009, San Jose, California, United States 


\title{
Ultrasound-modulated optical microscopy for ex vivo imaging of scattering biological tissue
}

\author{
Sri-Rajasekhar Kothapalli and Lihong V. Wang \\ Optical Imaging Laboratory, Department of Biomedical Engineering, Washington University in St. \\ Louis, Campus Box 1097, One Brookings Dr. St. Louis, Missouri, 63130
}

\begin{abstract}
Ultrasound-modulated optical microscopy (UOM) based on a long-cavity confocal Fabry-Perot interferometer (CFPI) [J.Biomed.Opt. 13(5), 0504046, (2008)] is used for real time detection of multiply scattered light modulated by high frequency $(30 \mathrm{MHz})$ ultrasound pulses propagating in an optically strongly scattering medium. In this article, we use this microscope to study the dependence of ultrasound-modulated optical signals on the optical absorption of objects embedded about $3 \mathrm{~mm}$ deep in tissue mimicking phantoms. These results demonstrate that the dependence is nearly linear. Most importantly, we imaged blood vasculature and melanin in highly scattering tissue samples from a mouse and a rat. Thus UOM can be used to study the morphology of blood vasculature and blood-associated functional parameters, such as oxygen saturation.
\end{abstract}

Keywords: microscopy, ultrasound-modulated optical tomography, absorption contrast, melanin, ultrasound, blood vasculature.

\section{INTRODUCTION}

\section{Motivation}

An impressive array of optical imaging modalities, such as optical coherence tomography (OCT); diffuse optical tomography (DOT); photoacoustic tomography (PAT), can now provide structural, functional, and molecular information about the scattering biological tissue based on various optical contrast mechanisms ${ }^{1-10}$. In addition, the technical advantages, such as non-ionizing radiation, ease of operation, low cost, and high sensitivity favor these technologies towards biomedical applications. Optical absorption and scattering of visible and near infrared light in soft biological tissue are related to tissue biochemical composition and morphology. The microscopic origin of the light absorption in tissue can be attributed to certain biomolecules (chromophores) - hemoglobin, melanin, water, and fat among others - that have strong and differential absorption at different visible and near infrared wavelengths. By exploiting differential absorption at a set of suitable visible or near infrared wavelengths, the concentrations of two forms of hemoglobin, oxyhemoglobin and deoxyhemoglobin, can be quantified. Using these concentrations, the saturation level of oxygen $\left(\mathrm{SO}_{2}\right)$, a physiologically important functional parameter, can be inferred. For example, tumor development usually involves hypoxia, a low level of $\mathrm{SO}_{2}$. Therefore, blood vasculature and $\mathrm{SO}_{2}$ can be readily mapped by modalities sensitive to optical absorption. ${ }^{11}$ On the other hand, the microscopic origin of light scattering in tissue is a result of complex manifestation of optical refractive index variations that are due to the various cellular structures and organelles and encodes morphologic and pathophysiologic changes in tissue. Thus, variations in scattering coefficient can provide information about neuronal activity ${ }^{12}$ and diseased tissue ${ }^{6}$. Optical imaging offers excellent intrinsic optical contrast but suffers from poor spatial resolution at depths beyond one transport mean free path in biological tissue due to strong scattering of light in biological tissue. On the other hand, ultrasound imaging offers excellent spatial resolution and imaging depth, both of which are scalable with ultrasound parameters. The higher the ultrasound frequency, the better the resolution of the image. However, the attenuation of ultrasound energy also increases with the frequency and this limits the penetration depth of the ultrasound pulse.

Many in vivo optical microscopy techniques offer micron-scale resolution at shallow (a few hundred $\mu \mathrm{m}$ ) imaging depths in the skin. Biological tissues, with the exception of ocular tissue, are highly scattering for light and singly backscattered light attenuates rapidly with the imaging depth. Thus these techniques are mainly based on the ballistic component of back reflected light for imaging while rejecting multiply scattered light. For example, confocal

Photons Plus Ultrasound: Imaging and Sensing 2009, edited by Alexander A. Oraevsky, Lihong V. Wang, Proc. of SPIE Vol. 7177, 71772N · C 2009 SPIE · CCC code: 1605-7422/09/\$18 · doi: 10.1117/12.810566 
microscopy can achieve $\sim 1 \mu \mathrm{m}$ resolution but can image up to only $0.5 \mathrm{~mm}$ into biological tissue. ${ }^{13-16}$ Opticalcoherence tomography (OCT) can achieve $\sim 10 \mu \mathrm{m}$ resolution but can image only $\sim 1 \mathrm{~mm}$ into scattering biological tissue. Therefore OCT has been quite extensively used for imaging ocular tissue or shallow regions of skin tissue.,17-22 Therefore they are fundamentally limited by penetration depth $(\sim 1 \mathrm{~mm})$, as light undergoes strong diffusion typically after passing one transport mean free path inside the scattering biological tissue. As many superficial lesions of interest are few $\mathrm{mm}$ deep inside scattering biological tissue, there is a need for development of new optical imaging modalities that can be of use in this imaging depth.

Ultrasound-mediated optical imaging modalities, Photoacoustic tomography (PAT) and Ultrasound-modulated optical tomography (UOT), are hybrid imaging modalities that combine advantages of both ultrasound and optical imaging to achieve high spatial resolution tomographic imaging using multiply scattered light. ${ }^{23}$ Therefore both the spatial resolution and the imaging depth of PAT and UOT are scalable using ultrasound parameters. Consequently, these modalities can be used both in macroscopic (deep tissue; up to several centimeters deep) as well as microscopic (several millimeters deep) regimes. In general, ultrasound transducers with low central frequencies $(1 \mathrm{MHz}-10 \mathrm{MHz})$ are used for deep tissue imaging while the transducers with high central frequency (20 MHz to $75 \mathrm{MHz}$ ) are used for microscopic imaging. Recently we demonstrated that microscopic imaging is feasible in ultrasound-modulated optical tomography (UOT) of soft biological tissues, using a high frequency focused ultrasound transducer with a $75 \mathrm{MHz}$ central frequency. Our experiments in tissue mimicking phantoms show that at an imaging depth of about $2 \mathrm{~mm}$, an axial resolution better than $30 \mu \mathrm{m}$ can be achieved, whereas the lateral resolution is $38 \mu \mathrm{m}$. This ultrasound-modulated optical microscopy (UOM) is based on confocal Fabry-Perot interferometer detection scheme.

In this Proceedings article, we use UOM to first study the dependence of ultrasound-modulated optical signal on optical absorption properties of objects embedded about $3 \mathrm{~mm}$ inside tissue mimicking phantoms. Our results on absorbing objects $\left(\mu_{a}<145 \mathrm{~cm}^{-1}\right.$ at $\left.532 \mathrm{~nm}\right)$ show that image contrast of absorbing objects is nearly linearly proportional to the absorption coefficient of the object. Most importantly, we use this microscopy to image blood vessels intact with tissue (such as ear and skin) samples of mice and rat. Our B-scan results show that images can be formed of the interior of blood vessels. Further we use maximum intensity projection (MIP) along the axial (depth) direction and show the structure of blood vessels. In addition we also image melanoma intact with the mice skin.

\subsection{Principle of ultrasound-modulated optical tomography (UOT)}

Ultrasound-modulated optical tomography (UOT) has been proposed to achieve high spatial resolution tomographic imaging using multiply scattered light. ${ }^{11,23}$ In this hybrid imaging modality, a focused ultrasonic wave modulates diffuse coherent light in scattering biological tissue. The detected modulated light is more sensitive than the measured nonmodulated light intensity to the local optical properties of the tissue within the ultrasound beam. ${ }^{24}$ Therefore, this imaging modality combines ultrasonic resolution and optical contrast. Moreover, mechanical contrast (due to acoustic impedance mismatch) of soft biological tissue can also be imaged by time-gating the modulated light. ${ }^{25}$ The physical mechanism of the modulation can be attributed to ultrasound-induced displacements of optical scatterers and to ultrasound-induced changes in the optical index of refraction. ${ }^{26-30}$ Since ultrasound waves consist of both compressions and rarefactions, each scattered photon passing through the ultrasound column acquires positive and negative phase shifts. Therefore the net phase accumulation along the total path length is relatively small. Moreover, the volume occupied by ultrasound inside the tissue is much smaller than the volume occupied by multiply scattered photons. Thus the volume of ultrasound and light interaction is relatively small compared to that of the non-modulated light. Consequently, various detection schemes have been proposed to detect ultrasound-modulated light efficiently, while other techniques were proposed to improve the imaging speed.

Marks et $a l^{30}$ first investigated the possibility of tagging diffused light with ultrasound. A CCD camera that provides parallel speckle detection was used to produce a better signal-to-noise ratio than a single square-law detector. ${ }^{31-33}$ Wang $e t a l^{34}$ developed ultrasound-modulated optical tomography and obtained images in tissue-simulating phantoms. Leutz and Maret ${ }^{35}$ analyzed ultrasonic modulation of light theoretically. Later Wang ${ }^{27}$ and Sakadžic ${ }^{28-29,36}$ used rigorous analytical models to explain the physical mechanisms associated with UOT. Leveque $e t a l^{31}$ developed a parallel speckle detection scheme that uses a source-synchronized lock-in technique in which a CCD camera works as a detector array. The signal-to-noise ratio (SNR) can be greatly improved by averaging the signals from all of the CCD pixels. This new technique allowed Leveque et al to obtain one-dimensional (1-D) images of buried objects in 
biological tissue. Yao and Wang obtained two-dimensional (2-D) images of multiple objects buried in biological tissue by using this technique. ${ }^{37}$ They also demonstrated that ultrasound-modulated diffuse light contributed to imaging. However, the spatial resolution along the ultrasonic axis is not as good as the lateral resolution in densely scattering biological tissue because of the elongated ultrasonic focal zone.

On the other hand, to obtain resolution along the ultrasonic axis, several groups of scientists explored various techniques, including an ultrasound frequency sweep ${ }^{38-39}$, computer tomography ${ }^{40}$, and tracking of ultrasound pulses ${ }^{41}$ or short bursts ${ }^{42}$. The pulsed ultrasound approaches provide direct resolution along the ultrasonic axis and are more compatible with conventional ultrasound imaging. They also reduced the imaging speed. Pulsed ultrasound can have a much higher instantaneous power than $\mathrm{CW}$ ultrasound, reducing the undesired effect of the increased noise owing to its wide bandwidth. Recently, Sakadzic $e t a l^{43}$ reported high-resolution ultrasound-modulated optical imaging with a longcavity confocal Fabry-Perot interferometer (CFPI). CFPI has a greater etendue, defined as the product of the acceptance solid angle and the area, than most CCD cameras and provides parallel speckle processing. Moreover, the current speed of CCD cameras is not sufficient for real time recording of the ultrasound-modulated light intensity produced by the ultrasonic pulses. In addition, a CFPI can detect the propagation of high-frequency ultrasound pulses in real time and tolerate speckle decorrelation. A CFPI is especially efficient at high ultrasound frequencies, where the background light can be filtered out effectively while the ultrasound modulated component is transmitted. They showed that optical features of $100 \mu \mathrm{m}$ in size embedded more than $3 \mathrm{~mm}$ below the surface of chicken breast tissue can be resolved with high contrast in both the axial and the lateral directions. At present, detection schemes based on photo-refractive crystals $^{44,45}$, Fabry-Perot interferometers ${ }^{43,46}$, and hole-burning crystals ${ }^{47}$ are the most suitable choices for fast parallel speckle detection.

\section{EXPERIMENT}

\section{Experimental setup: Scanning confocal Fabry-Perot interferometer based detection technique}

The details of the UOM experimental setup (Fig.1) based on CFPI (50-cm cavity length, $0.1 \mathrm{~mm}^{2}$ sr etendue, and $\sim 20$ finesse) have been described earlier. ${ }^{43,46}$ The CFPI was as a real time optical filter, for filtering multiply scattered light modulated by high frequency ultrasound pulses propagating in biological tissue. Figure 1 shows the configuration of the sample $\mathrm{S}$ that is imaged, as well as the oblique configuration of the light illumination and ultrasound insonification of the sample S. The ultrasonic and optical beams have the same focus below the sample surface. A high frequency focused ultrasound transducer with central frequency of $30 \mathrm{MHz}$ (Panametrics, $4.25 \mu$ s fused silica delay lines, 0.25 inch element sizes, $5.5 \mathrm{~mm}$ focal lengths, $80 \%$ estimated fractional bandwidths) was used in our experiments. This focused transducer is modified from a commercial broadband delay-line transducer (V213-BB-RM, Panametrics) by grinding a concave spherical lens directly in its quartz delay line (Fig. 1b). This concave lens works as a focusing acoustic lens. The lateral resolution of UOM in biological tissues is mainly determined by the center frequency and the numerical aperture (NA) of the ultrasound transducer, and the axial resolution is primarily determined by the frequency bandwidth of the ultrasound transducer. The center frequency also limits the imaging depth due to the frequencydependent ultrasonic attenuation, $0.7-3 \mathrm{~dB} /(\mathrm{cm} / \mathrm{MHz})$ in human skin. Our earlier experiments, using ultrasound transducer with the $30 \mathrm{MHz}$ central frequency, in tissue mimicking phantoms show that an axial resolution of about 60 $\mu \mathrm{m}$ and the lateral resolution of $70 \mu \mathrm{m}$ can be achieved in a depth range of about $2.5 \mathrm{~mm} .{ }^{46}$ The transducer is driven by the square bipolar pulse with period of $34 \mathrm{~ns}$. This bipolar pulse is generated by the trigger generator (Stanford Research, DG535) and amplified with an amplifier (Amplifier Research, 75A250). The focal ultrasound pressure measured by the needle hydrophone (ONDA HNV-0200) was about $3 \mathrm{MPa}$, within the ultrasound safety limit at these frequencies for tissues without well-defined gas bodies. ${ }^{48,49}$

In the experiment, the laser light (Coherent, Verdi; 532-nm wavelength) was focused onto a spot of $\sim 100 \mu \mathrm{m}$ in diameter below the surface of an otherwise scattering-free sample. The optical power delivered to the sample was about $50 \mathrm{~mW}$. However, for in-vivo experiments the duration of the light exposure to the living subject can be reduced to only a few $\mu$ s for each cycle of ultrasound propagation through the region of interest, to meet the ANSI safety limit for average power. ${ }^{50}$ Diffusely reflected light was collected by a multi-mode optical fiber with a $600 \mu \mathrm{m}$ core diameter. The axes of propagation of the ultrasonic and optical beams are denoted by $X$ and $Y$, respectively. The sample was mounted on a three-axis $(X 1, Y 1$, and $Z 1)$ motorized translational stage and controlled by LabView program. The ultrasound transducer UT and the sample S were immersed in water for acoustic coupling, and thus the light focusing optics FO, 
and the collecting fiber CF were also immersed in the same water tank. The collected light was coupled into the CFPI, using coupling optics $\mathrm{CO}$, and was operated in transmission mode. The filtered light sampled by the beam sampler (5:95) and detected by the photodiode PD (New Focus Model\#2031) was used in a cavity alignment procedure. The cavity was first swept through one free spectral range to find the position of the central frequency of the unmodulated light. Then, CFPI mirror M2 was displaced by a calibrated amount so that the cavity was tuned to the frequency of one sideband of the ultrasound-modulated light $(30 \mathrm{MHz}$ greater than the laser light frequency). The filtered light transmitted by the beam sampler was detected by an avalanche photo-diode (APD) (Advanced Photonix) and the signal was sampled at $200 \mathrm{Ms} / \mathrm{sec}$ with a data acquisition board (GAGE, CS14200). A LabView computer program controlled the movement of the CFPI mirror and the other sequences of the control signals. A trigger generator (Stanford Research, DG535) triggered both the ultrasound pulse generation and the data acquisition from the APD. Since the resonant frequency of the CFPI cavity coincided with one sideband of the ultrasound-modulated light, the signal acquired by the APD during the ultrasound propagation through the sample represented the distribution of the ultrasound-modulated optical intensity along the ultrasonic axis and, therefore, yielded a one-dimensional (1D) image (A-line) along the $X$ direction. In each operational cycle, the resonant frequency of the CFPI was tuned first, and then data from 4000 ultrasound pulses were acquired in one second. Averaging of 3 to 5 cycles was usually needed to obtain a satisfactory SNR for each 1D image. Two-dimensional images were obtained by scanning the sample along the $Z$ direction and acquiring each corresponding 1D image.

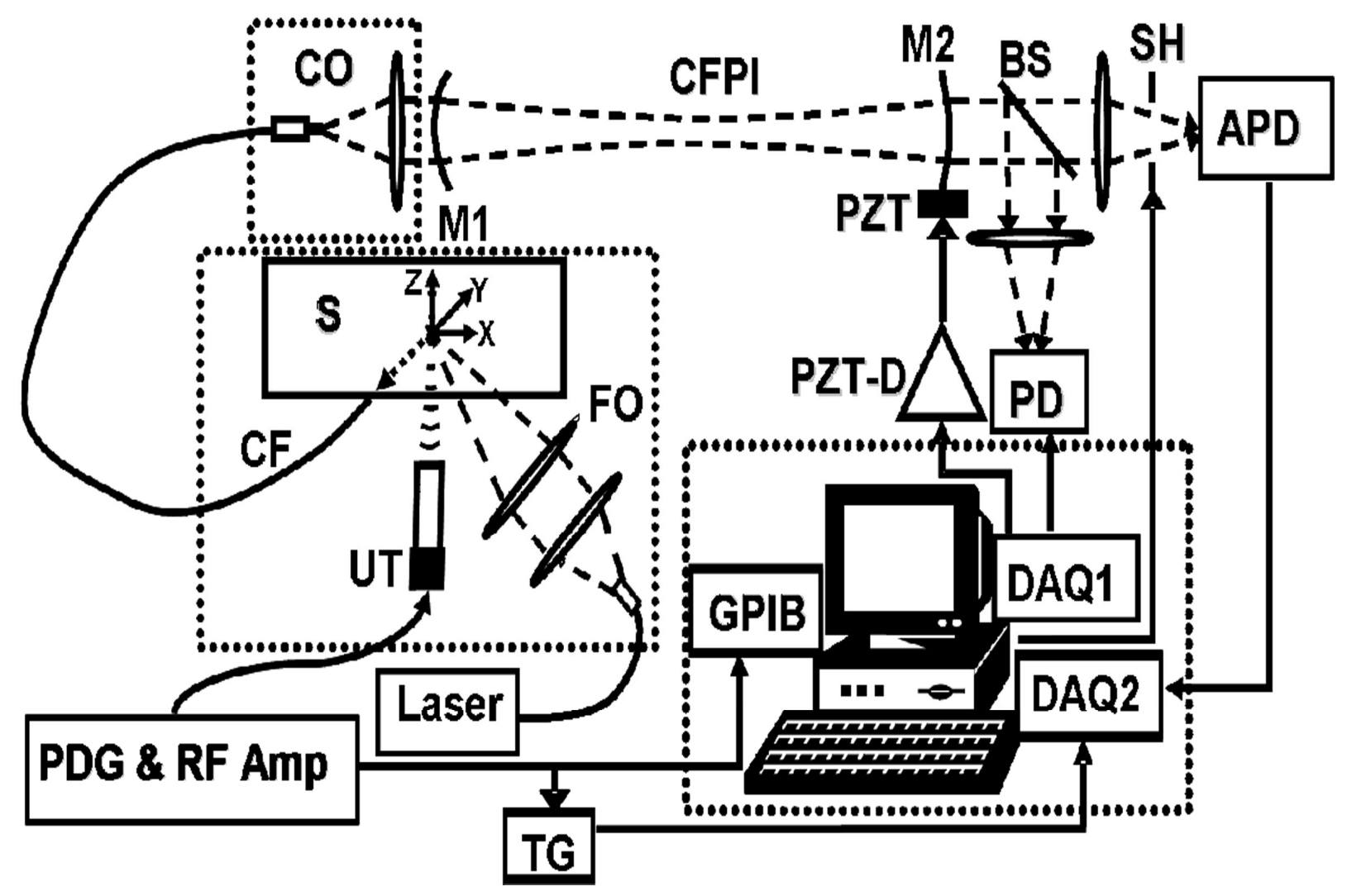

Figure 1: Schematic of the experimental setup: APD, avalanche photodiode; BS, beam splitter; CF, collecting fiber; CFPI, confocal Fabry-Perot interferometer; CO, coupling optics; DAQ, data acquisition board; FO, focusing optics; M1,M2, Mirrors inside the cavity; PD, photodetector; PDG, pulse delay generator; PZT, piezoelectric transducer; PZT-D, piezoelectric transducer driver; RF Amp, RF amplifier; S, sample; SH, shutter; TG, trigger generator; UT, ultrasonic transducer;

After each B-scan along the $x$ axis, the sample was returned to its original $x$ coordinate and further translated by one scanning step (usually $0.1 \mathrm{~mm}$ ) along $y$ axis to start a new B-scan. Thus several B-scans were obtained by raster scanning the sample in the $\mathrm{x}-\mathrm{y}$ plane. To obtain high contrast blood vessel imaging, each B-scan is processed, by 
subtracting each A-line in B-scan from an A-line obtained from the transparent region of the respective B-scan. We further perform maximum intensity projection (MIP), along the $z$ axis, on each of the processed B-scan images. The final maximum intensity projection (MIP) image was obtained by combining projections from all B-scans.

\subsection{Phantom preparation}

Ultrasound-modulated optical imaging is primarily aimed at imaging intrinsic optical contrast of soft biological tissue. Therefore, we prepared several objects of different absorption and measured the ultrasound-modulated optical signal with our UOM setup presented in Figure 1. The four objects are made of $15 \%$ porcine gelatin, with different amounts of the Trypan Blue dye, such that optical absorption coefficient at $532 \mathrm{~nm}$ are $145 \mathrm{~cm}^{-1}, 109 \mathrm{~cm}^{-1}, 72 \mathrm{~cm}^{-1}, 36$ $\mathrm{cm}^{-1}$ and $0.1 \mathrm{~cm}^{-1}$ (the whole blood absorption at $532 \mathrm{~nm}$ is around $120 \mathrm{~cm}^{-1}$ ). The approximate dimensions of all four objects are $2 \mathrm{~mm}, 2 \mathrm{~mm}, 1 \mathrm{~mm}$ in the X, Y, and Z directions. All four objects were about $1 \mathrm{~mm}$ thick in ultrasound propagation direction. The widths (along the $Z$-axis) of the objects were perpendicular to the ultrasound beam. These objects were transparent enough for the ultrasound, and absorptive for the light. The background tissue phantom is made from gelatin, water and 1\% Intralipid (20\% Liposyn II, Intravenous Fat Emulsion Hospira, Inc.) with a 1-mm optical transport mean free path. The tissue phantom is an optically scattering slab $10 \mathrm{~cm}$ wide in the $\mathrm{Y}$ (ultrasound scanning axis) and $\mathrm{Z}$ directions and $7 \mathrm{~mm}$ thick in the $\mathrm{X}$ direction (light propagation direction). The optical reduced scattering and absorption coefficients of the scattering slab (background medium) are $10 \mathrm{~cm}^{-1}$ and $0.1 \mathrm{~cm}^{-1}$, respectively. As shown in the Figure 2 a piece of stainless steel needle $(260 \mu \mathrm{m}$ in outer diameter) and all four objects were placed inside the scattering slab at a depth of $X=3 \mathrm{~mm}$. Since ultrasound waves are strongly reflected by the needle, it helped in aligning the resolution targets precisely at the ultrasound focal point. We used a pulser (GE Panametrics, 5072PR) in this alignment procedure.

Preparation of tissue samples: We obtained several tissue samples such as ear and skin from dead (sacrificed during other experiments in our lab) mice and rat. As shown in Figures 3(a) and 4(a), a piece of stainless steel needle (260 $\mu \mathrm{m}$ in diameter) and these tissue samples were then placed inside an optically clear medium (made from gelatin and water) at a depth of $\mathrm{X}=2.5 \mathrm{~mm}$. This clear medium is $10 \mathrm{~cm}$ wide in the $\mathrm{Y}$ (ultrasound scanning axis) and $\mathrm{Z}$ directions and 5 $\mathrm{mm}$ thick in the $\mathrm{X}$ direction (light propagation direction).

\section{RESULTS AND DISCUSSION}

\subsection{Ultrasound-modulated optical signal dependence on optical properties}

The contrast in ultrasound-modulated optical imaging is primarily based on optical properties of the biological tissue. Although both scattering and absorption influence the strength of the signal, variations of the ultrasound-modulated intensity are more sensitive to absorption. ${ }^{24,51-52}$ Therefore it is of primary importance to explore the dependence of the ultrasound-modulated optical signal on optical properties using the UOM system shown in Figure 1. As described in the previous section, we prepared optically scattering sample and embedded four optically absorbing objects (prepared using a mixture of gelatin, water and Trypan Blue dye) $3.0 \mathrm{~mm}$ below the surface of the sample. A photograph of this sample is shown in Fig. 3 (a). Figure 3.2(b) shows a background subtracted two-dimensional (B-scan) image of all four objects shown in Fig. 3.2 (a), obtained by scanning the sample in the $X$ direction. Respective 1-D profiles (A-lines) along the $Z$ axis through the center of all four objects are plotted in Fig. 3.2(c). As shown in Figures 3(c) both the width and height of the signal drop, decreases with decrease in absorption coefficient of the object. This decrease in width of the signal drop can be attributed to increase in diffusion of photons (into the object) with decrease in absorption coefficient of the object. The decrease in height of the signal drop with increase in absorption coefficient of the object can be directly related to increase in absorption of photons.

The measured contrast at the center of all three absorbing objects in Fig. 3(b), demonstrates that the contrast of each object was nearly linearly related to the absorption coefficient of the object. More importantly, the measurable absorptions are clearly much lower than the blood absorption, which implies that this technology has a great potential in detecting small blood vessels and other functional parameters associated with blood such as oxygenation saturation. This linear dependence can be explained analytically. Simple theoretical considerations show that in the limit of a small 
absorption compared to the path length of the light through the absorbing object, the dependence of ultrasoundmodulated signal intensity on the object absorption should be nearly linear. ${ }^{51}$
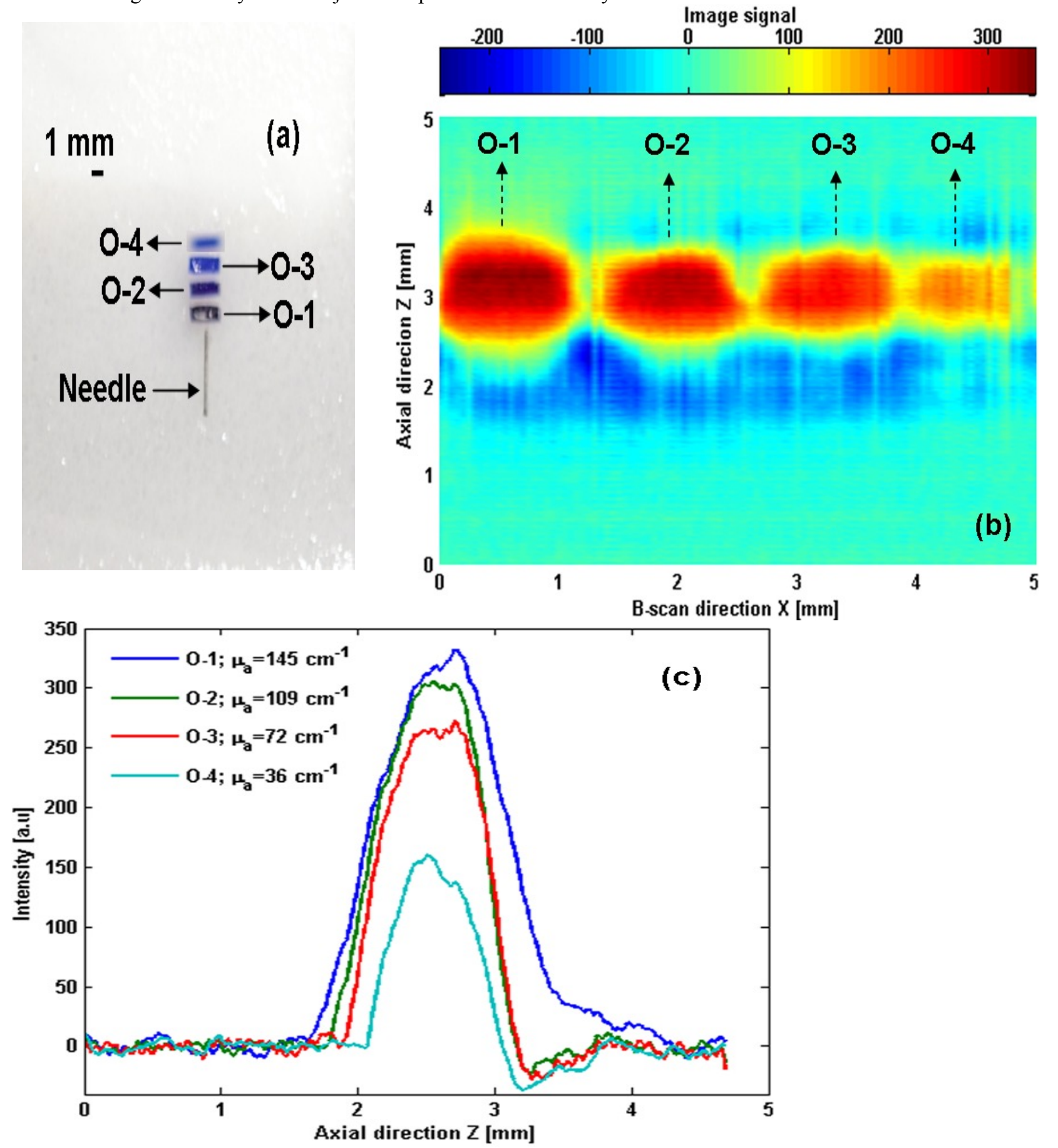

Fig.2. Dependence of ultrasound-modulated optical signal on optical properties. (a) Photograph of four optically absorbing objects $\left(\mu_{a}=145 \mathrm{~cm}^{-1}, \mu_{a}=109 \mathrm{~cm}^{-1}, \mu_{a}=72 \mathrm{~cm}^{-1}, \mu_{a}=36 \mathrm{~cm}^{-1}\right.$, and all four having the same $\mu_{s}^{\prime}=0.5 \mathrm{~cm}^{-1}$ ) placed vertically below a piece of needle, respectively. These four objects are marked as O-1, O-2, O-3, and O-4 respectively. (b) Background subtracted B-scan UOM image of the sample shown in (a). (c) Respective 1-D profiles (A-lines) along the $Z$ axis through the center of all four objects. 
If the modulation of the light intensity is expressed as $M\left(\mu_{a}\right)$ where $\mu_{a}$ is the optical absorption coefficient of the object inside the tissue, then we can model the dependence of the $M\left(\mu_{a}\right)$ on the probability density function of the photon pathlength $f(p)$ as

$$
M\left(\mu_{a}\right)=\int \bar{M}(p) \exp \left[-\mu_{a} p\right] f(p) d p
$$

where, $M(p)$ is the modulated light intensity along the path of length $p$, which does not include absorption. When the absorption is slightly increased to $\mu_{a 1}=\mu_{a}+\Delta \mu_{a}$, the difference in modulation intensities, $\Delta M=M\left(\mu_{a 1}\right)-M\left(\mu_{a}\right)$, can be expressed as:

$$
\Delta M=\int \bar{M}(p) \exp \left[-\mu_{a} p\right] f(p)\left\{\exp \left[\left(\mu_{a}-\mu_{a 1}\right) p\right]-1\right\} d p
$$

If we assume that $\mu_{a} p$ is less than unity, in the case of objects of small dimensions in biological tissue (like small blood vessels and capillaries), we obtain

$$
\Delta M \approx-\Delta \mu_{a} \int \bar{M}(p) \exp \left[-\mu_{a} p\right] f(p) p d p
$$

i.e. change in ultrasound-modulated light intensity depends linearly on the variations in object absorption.

\subsection{Ex-vivo imaging using tissue samples}

\subsubsection{Imaging blood vasculature intact with rat ear}

Now we explore the potential of UOM for imaging intrinsic optical absorption due to blood using tissue sample (ear) obtained from the dead rat. As described in the phantom preparation section, these optically scattering tissue samples are placed in a clear medium. Figure 4(a) shows a photograph of blood vasculature inside a rat ear. Based on this photograph, the imaged vessels are $\sim 100 \mu \mathrm{m}$ in diameter and the ear is about $350 \mu \mathrm{m}$ thick. A piece of stainless steel (outer diameter $250 \mu \mathrm{m}$ ) is used to align the focal plane of the ultrasound transducer to the plane of ear placed inside a 5 $\mathrm{mm}$ thick clear medium. Figure 4(b) shows typical UOM B-scan image. As discussed in the previous sections, B-scan image is a 2-D cross-sectional (along the z-x plane) image obtained by scanning the sample along the $x$ direction and acquiring each corresponding 1-D image (A-line) with identical $y$ coordinates. Each B-scan took about 8 minutes (100 A-lines, $20 \mu \mathrm{m}$ step size, and $5 \mathrm{~s} / \mathrm{A}$-line) to complete. For each A-line, the time of propagation is multiplied by 1500 $\mathrm{ms}^{-1}$, the approximate speed of sound in the sample, to be converted to distance (depth) along the $z$ axis. Therefore, the vertical axis of B-scan image represents the depth from the surface of the ear and the horizontal axis represents position of the transducer along the $x$ (B-scan) direction. The B-scan shows the perpendicular (to the imaging plane of B-scan) crossection of blood vessel, which is nearly circular in shape. From these B-scan images (also from results on absorbing objects in Figure 3 (b)) we can infer that UOM has the potential to image interior of blood vessel. Size of the vessel is $0.1 \mathrm{~mm}$ (5 pixels) in the image. After each B-scan along the $x$ axis, the sample was returned to its original $x$ coordinate and further translated by one scanning step $(0.1 \mathrm{~mm})$ along $y$ axis to start a new B-scan Thus by raster scanning the sample in the $\mathrm{x}-\mathrm{y}$ plane we obtained total of $40 \mathrm{~B}$-scans.

We subtracted each A-line in B-scan from an A-line obtained from the transparent region of the respective B-scan, to obtain high contrast blood vessel imaging. A typical processed B-scan image is shown in Figure 3(b). In addition, we performed maximum intensity projection (MIP), along the $z$ axis, on each of the processed B-scan images. The final maximum intensity projection (MIP) image, Figure 3 (c), was obtained by combining projections from all B-scans. Good agreement in the vascular structure can be observed between the projection image (Figure 3(c)) and the photograph (Figure 3(a)). 


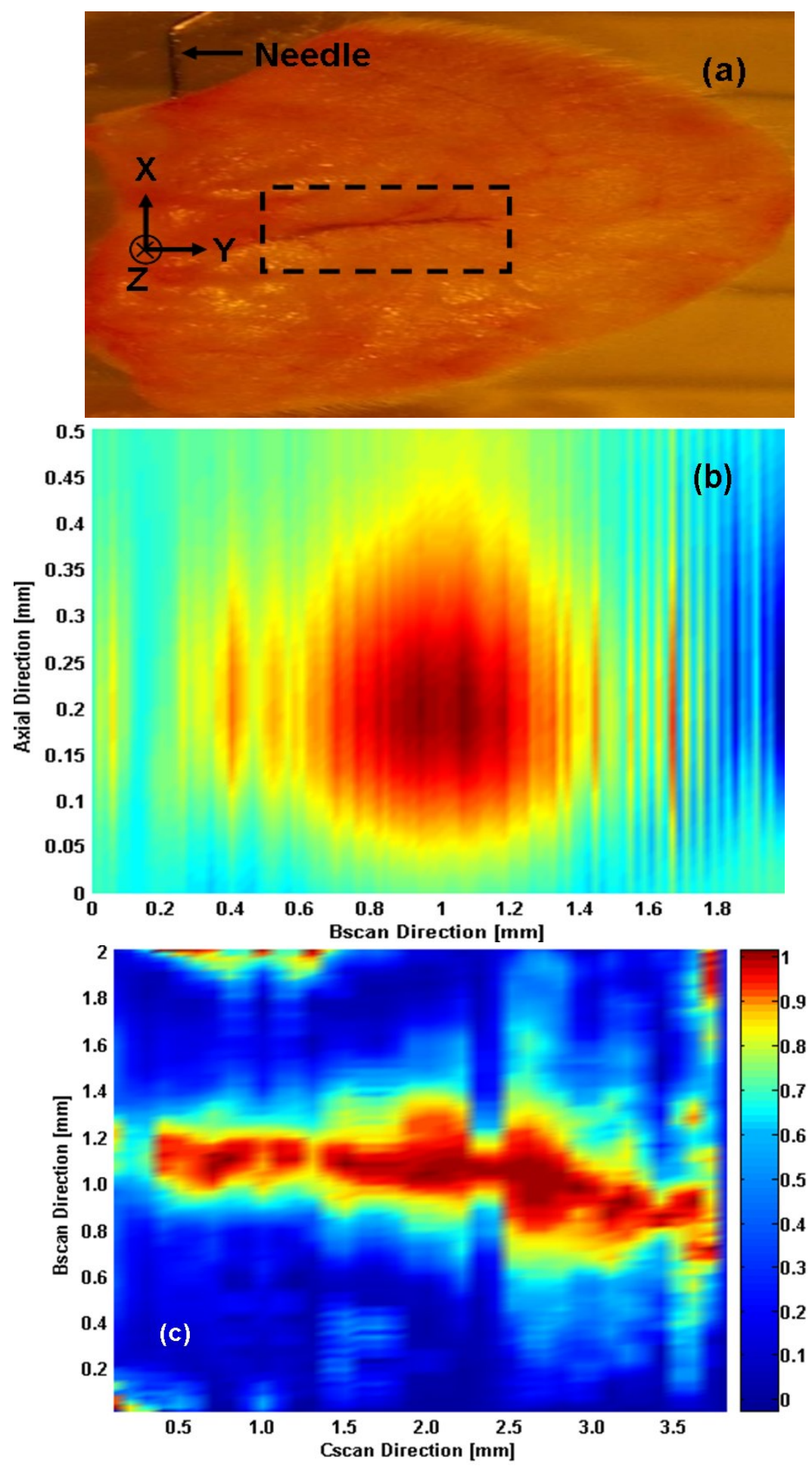

Fig. 3. Imaging intact blood vasculature in a rat ear. (a) Photograph of blood vasculature inside the rat ear. Boxed region shows the region raster scanned. (b). Typical two dimensional B-scan image. (c) Maximum intensity projection (MIP) image of (a). 


\subsubsection{Imaging skin melanoma}

The incidence of melanoma is dramatically increasing than other cancer related diseases. ${ }^{53}$ The depth and the thickness of the melanoma affected cancerous tissue are particularly important in the choice of the treatment. Figure 4 (a) shows the photograph of the skin sample obtained from the dead mice. This mouse was inoculated with highly invasive B16 skin melanoma cells (ATCC, Manassas, VA, USA) subcutaneously in the upper lumbar area to the left of the vertebra four days before the experiment. This mouse was sacrificed during other experiments in our lab. In a tissue sharing program we obtained the skin sample from this mouse.
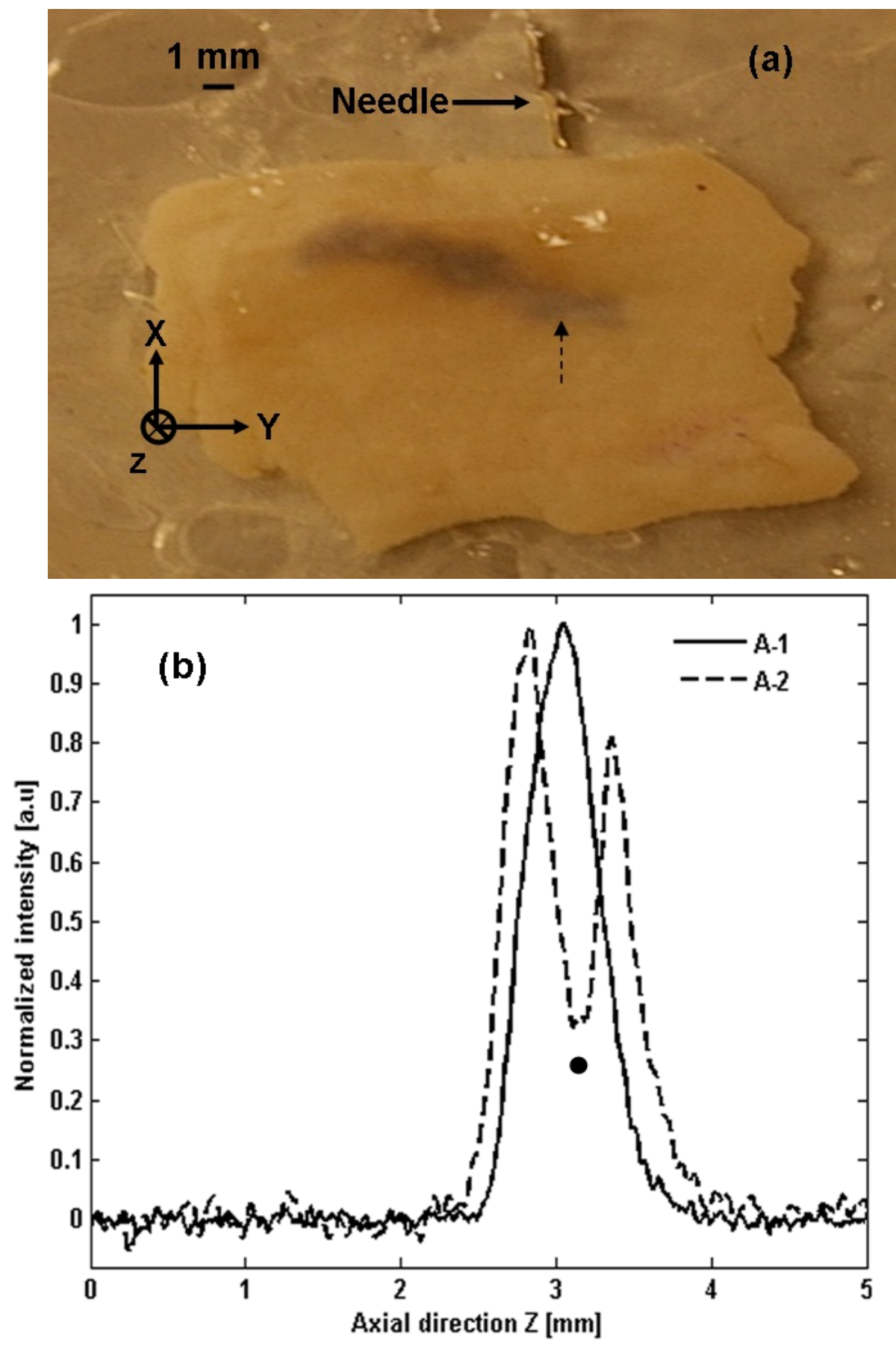

Proc. of SPIE Vol. $717771772 \mathrm{~N}-9$ 


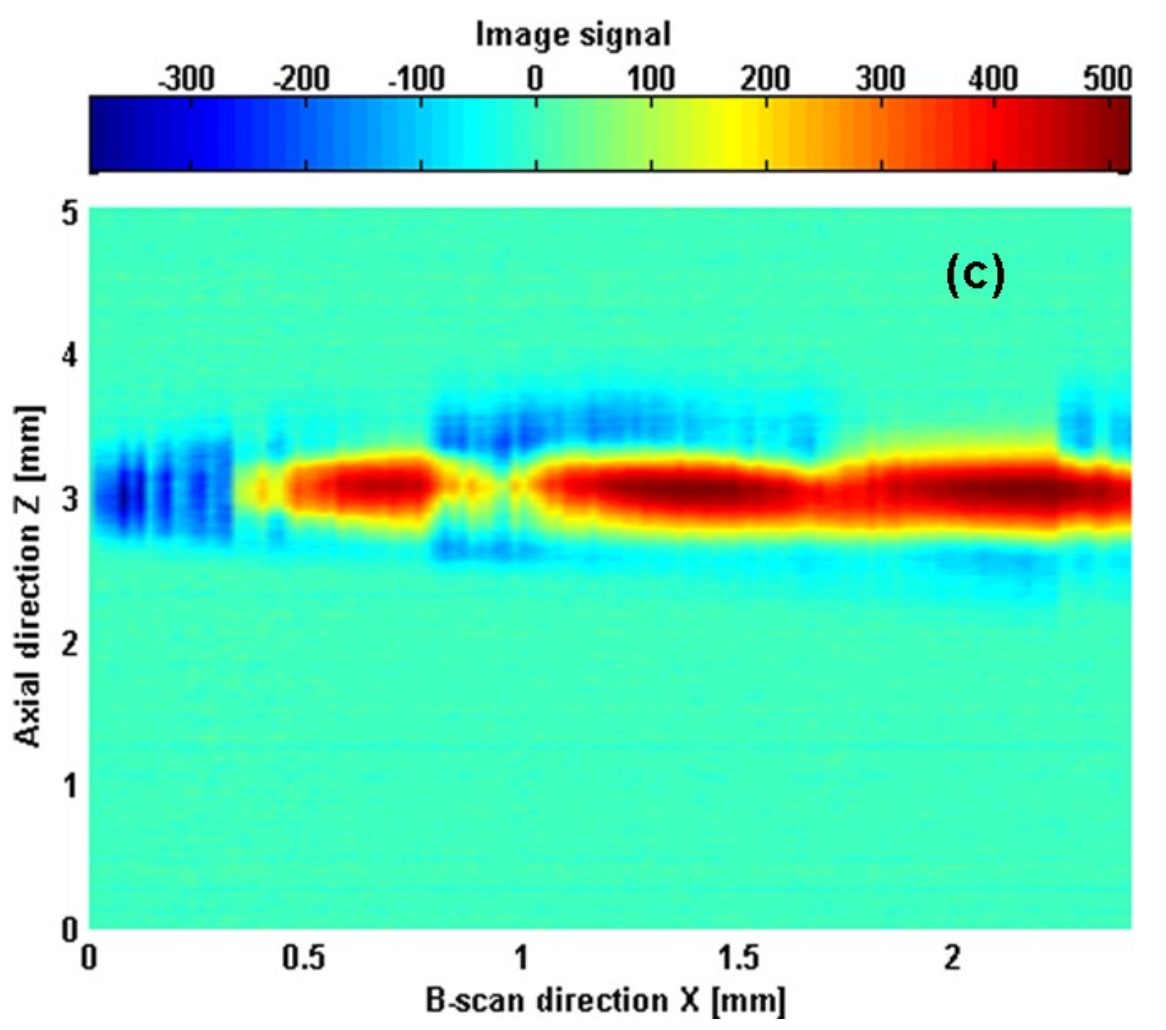

Fig. 4. Imaging intact skin melanoma. (a) Photograph of intact skin melanoma obtained from sacrificed mouse. (b) A-line, A-1, represents temporal dependence of ultrasound-modulated light intensity during the propagation of ultrasound pulse through the ear tissue sample. A-2 represents typical A-line passing through the melanin. A dot placed below this curve in the figure represents the position of the melanoma along the ultrasound propagation direction. (c) Typical two dimensional B-scan image along the dotted arrow shown in (a).

Fig 4 (a) shows melanin intact with the skin. Figure 4 (b) shows a typical normalized A-line (A-2) from the original data, passing through the melanin located at about $300 \mu \mathrm{m}$ deep in the skin tissue sample. A dot placed below this represents the position of the melanoma along the ultrasound propagation direction (axial direction or depth direction). The local drop in the signal strength at the position of melanoma is primarily due to ultrasound-modulated optical absorption of melanin. A-1 represents A-line not passing through the melanoma. Figure 4(c) shows typical B-scan image along the dotted arrow of Figure 4(a)) obtained using UOM. Each B-scan took about 4 minutes (50 A-lines, 50 $\mu \mathrm{m}$ step size, and $5 \mathrm{~s} / \mathrm{A}$-line). From these results the maximum thickness of the melanoma of about $0.5 \mathrm{~mm}$ is measured.

\section{CONCLUSIONS}

In summary, ultrasound-modulated optical microscopy is used for ex vivo imaging of blood vasculature intact with rat ear and imaging melanoma intact with mouse skin. This study and our previous studies ${ }^{52}$ demonstrated feasibility of blood vasculature imaging using ultrasound-modulated optical microscopy. This suggests that ultrasound-modulated optical imaging can be potentially be used for functional imaging of living subjects. This study might be compatible with other imaging modalities, like photoacoustic tomography, in reconstructing optical properties of biological tissue.

\section{ACKNOWLEDGEMENT}

This research was supported by the National Institutes of Health grants R01 CA094267 and R33 CA106728 


\section{REFERENCES}

[1] Massoud, T. F., and Gambhir, S.S., "Molecular imaging in living subjects: seeing fundamental biological processes in a new light," Genes and Development 17, 545-580 (2003).

[2] Weissleder, R. and Ntziachristos, V., "Shedding light onto live molecular targets," Nat. Med. 9, 123-128 (2003).

[3] Boas, D.A., Brooks, D.H., Miller, E.L., DiMarzio,C.A., Kilmer,M., Gaudette,R.J. and Zhang,Q., "Imaging the body with diffuse optical tomography," IEEE Sig. Proc. Nov, 18, 57-75 (2001).

[4] Fujimoto, J. G., "Optical coherence tomography for ultrahigh resolution in vivo imaging," Nat. Biotech. 21, 13611367 (2003).

[5] Zhang, H. F., Maslov,K., Stoica, G. and L.-H. Wang, "Functional photoacoustic microscopy for high-resolution and noninvasive in vivo imaging," Nat. Biotech. 24, 848-851 (2006).

[6] Srinivasan, S., Pogue, B. W., Jiang, S., Dehghani, H. Kogel,C., Soho, S., Gibson, J. J., Tosteson, T. D., Poplack S. P. and Paulsen, K. D. "Interpreting hemoglobin and water concentration, oxygen saturation and scattering measured in vivo by near-infrared breast tomography," Proc. Natl. Acad. Sci. 100, 12349-12354 (2003).

[7] Inoue, H., Kudo S., and Shiokawa,A., "Laser-scanning confocal microscopy and endocytoscopy for cellular observation of the gastrointestinal tract," Nat. Clin. Pract Gastroenterol. Hepatol. 2, 31-37 (2005).

[8] Gurden, H., Uchida N., and Mainen,Z., "Sensory-Evoked Intrinsic Optical Signals in the Olfactory Bulb Are Coupled to Glutamate Release and Uptake," Neuron 52, 335 - 345 (2006).

[9] Aitken, P. G., Fayuk, D., Somjen G. G., and Turner, D. A., "Use of intrinsic optical signals to monitor physiological changes in brain tissue slices," Methods 18, 91-103 (1999).

[10] Inoue, H., S. Kudo and A. Shiokawa, "Laser-scanning confocal microscopy and endocytoscopy for cellular observation of the gastrointestinal tract," Nat. Clin. Pract Gastroenterol. Hepatol. 2, 31-37 (2005).

[11] Wang, L. V. and Wu, H.-i., Biomedical Optics: Principles and Imaging Wiley, New York, 2007.

[12] Aguirre, A. D., Chen Y., Fujimoto,J.G., Ruvinskaya, L., Devor, A. and Boas,D. A., "Depth-resolved imaging of functional activation in the rat cerebral cortex using optical coherence tomography," Opt. Lett. 31, 3459-3461 (2006).

[13] Wilson, T., ed., Confocal microscopy (Academic Press, New York, 1990).

[14] Pawley, J. B., ed., Handbook of biological confocal microscopy (Plenum Press, New York, 1990).

[15] Nie, S., Chiu,D. T., and Zare,R. N., "Probing individual molecules with confocal fluorescence microscopy," Science 266, 1018-21 (1994).

[16] Rajadhyaksha, M., Anderson,R. R. and Webb,R. H., "Video-rate confocal scanning laser microscope for imaging human tissues in vivo," Appl. Opt. 38, 2105-2115 (1999).

[17] Huang, D., Swanson, E. A., Lin,C. P., Schuman, J. S., Stinson,W. G., Chang,W., Hee,M. R., Flotte,T., Gregory, K., Puliafito, C. P., and Fujimoto,J. G., "Optical Coherence Tomography," Science 254, 1178-1181 (1991).

[18] Fercher, A. F., "Optical coherence tomography," J. Biomed. Opt. 1, 157-173 (1997).

[19] Rollins,A., Yazdanfar, S., Kulkarni, M., Ung-arunyawee,R., and Izatt,J. A., "In vivo video rate optical coherence tomography," Opt. Exp. 3, 219-229 (1998).

[20] Pan, Y. T., and Farkas, D. L., "High-resolution imaging of living human skin with optical coherence tomography," Scanning 21, 134-135 (1999).

[21] De Boer, J. F., Milner,T. E. and Nelson, J. S., "Determination of the depth-resolved Stokes parameters of light backscattered from turbid media by use of polarization-sensitive optical coherence tomography," Opt. Lett. 24, 300-302 (1999).

[22] Zhao,Y. H., Chen,Z. P., Saxer,C., Xiang,S. H., de Boer,J. F., and Nelson,J. S.,"Phase-resolved optical coherence tomography and optical Doppler tomography for imaging blood flow in human skin with fast scanning speed and high velocity sensitivity," Opt. Lett. 25, 114-116 (2000).

[23] Wang, L.-H., "Ultrasound-mediated biophotonic imaging: a review of acousto-optical tomography and photoacoustic tomography," J. of Dis. Mark. 19, 123-138 (2004).

[24] Kothapalli, S.-R., Sakadzic,S., Kim, and Wang,L. V. "Imaging optically scattering objects with ultrasoundmodulated optical tomography," Opt. Lett. 32, 2351-2353 (2007).

[25] Xu, X., Zhang,H., Qing,D., Kim,C., Hemmer,P., Wang, L. V., "Photorefractive detection of tissue optical and mechanical properties by ultrasound modulated optical tomography," Opt. Lett. 32, 656-658 (2007).

[26] Leutz, W. and Maret, G., "Ultrasonic modulation of multiply scattered-light," Physica B 204, 14-19 (1995).

[27] Wang, L. V., "Mechanisms of ultrasonic modulation of multiply scattered coherent light: an analytic model," Phys.

Rev. Lett. 87, 043903-(1-4) (2001). 
[28] Sakadzic, S. and Wang,L. V., "Ultrasonic modulation of multiply scattered coherent light: an analytical model for anisotropically scattering media," Phys. Rev. E 66, 026603-(1-9) (2002).

[29] Sakadzic, S. and Wang, L. V., "Modulation of multiply scattered coherent light by ultrasonic pulses: Analytical model," Phys. Rev. E 72, 036620-(1-12) (2005).

[30] Marks, F. A., Tomlinson,H. W. and Brooksby,G. W. "A comprehensive approach to breast cancer detection using light: photon localization by ultrasound modulation and tissue characterization by spectral discrimination," Proc. SPIE $1888,500-510$ (1993).

[31] Leveque, S., Boccara,A. C., Lebec, M., and Saint-Jalmes,H. "Ultrasonic tagging of photon paths in scattering media: parallel speckle modulation processing," Opt. Lett. 24, 181-183 (1999).

[32] Yao, G., Jiao,S.-L. and Wang,L. V., "Frequency-swept ultrasound-modulated optical tomography in biological tissue by use of parallel detection," Opt. Lett. 25, 734-736 (2000).

[33] Gross, M., Goy, P. and M. Al-Koussa, "Shot-noise detection of ultrasound-tagged photons in ultrasound modulated optical imaging," Opt. Lett. 28, 2482-2484 (2003).

[34] Wang, L. V., Jacques,S. L. and Zhao,X., "Continuous-wave ultrasonic modulation of scattered laser light to image objects in turbid media," Opt. Lett. 20, 629-631 (1995).

[35] Leutz, W. and Maret,G., "Ultrasonic modulation of multiply scattered-light," Physica B 204, 14-19 (1995).

[36] Sakadžić, S. and Wang,L. V., "Correlation transfer and diffusion of ultrasound-modulated multiply scattered light," Phys. Rev. Lett. 96, 0163902-(1-4) (2006).

[37] Yao, G., and Wang, L.-H.,"Theoretical and experimental studies of ultrasound-modulated optical tomography in biological tissue," Appl. Opt. 39, 659-664 (2000).

[38] Wang, L. V. and Ku, G., "Frequency-swept ultrasound-modulated optical tomography of scattering media," Opt. Lett. 23, 975-977 (1998).

[39] Yao, G., Jiao, S.-L. and Wang,L. V., "Frequency-swept ultrasound-modulated optical tomography in biological tissue by use of parallel detection," Opt. Lett. 25, 734-736 (2000).

[40] Li, J. and Wang, L. V., "Ultrasound-modulated optical computed tomography of biological tissues," Appl. Phys. Lett. 84, 1597-1599 (2004).

[41] Hisaka, M., Sugiura,T. and Kawata,S. "Optical cross-sectional imaging with pulse ultrasound wave assistance," J. Opt. Soc. Am. A-Opt. Image Sci. Vis. 18, 1531-1534 (2001).

[42] Lev, A. and Sfez, B. G., "Pulsed ultrasound-modulated light tomography," Opt. Lett. 28, 1549-1551 (2003).

[43] Sakadzic S. and Wang,L. V. "High-resolution ultrasound-modulated optical tomography in biological tissues," Opt. Lett. 29, 2770-2772 (2004).

[44] Murray, T. W., Sui,L., Maguluri,G., Roy,R., A., Nieva,A., Blonigen, F. and DiMarzio,C. A., "Detection of ultrasound modulated photons in diffuse media using the photorefractive effect," Opt. Lett. 29, 2509-2511 (2004).

[45] Rousseau, G., Blouin,A. and Monchalin,J., "Ultrasound-modulated optical imaging using a powerful long pulse laser," Opt. Express 16, 12577-12590 (2008).

[46] Kothapalli, S.-R. and Wang,L. V. "Ultrasound-modulated optical microscopy," J. Biomed. Opt. 13, 0504046-(18)(2008).

[47] Li,Y., Zhang,H., Kim,C. Wagner, K. H.,Hemmer,P. and Wang,L. V., "Pulsed ultrasound-modulated optical tomography using spectral-hole burning as a narrowband spectral filter," Appl. Phys. Lett. 93, 011111-(1-3) (2008).

[48] AIUM, http://www.aium.org/, Mammalian in Vivo Ultrasonic Biological Effects, 1992.

[49] Duck, F. A., "Medical and non-medical protection standards for ultrasound and infrasound," Prog. Biophys. Mol. Biol. 93, 176-191 (2007).

[50] Laser Institute of America, American National Standard for the Safe Use of Lasers ANSI Z136.1-2000 (ANSI, Orlando, Florida, (2000).

[51] Yao, G. and Wang, L. V., "Signal Dependence and Noise Source in Ultrasound-Modulated Optical Tomography," Appl. Opt. 43, 1320-1326 (2004).

[52] Kothapalli, S.-R. and Wang, L. V., "Ex vivo blood vessel imaging using ultrasound-modulated optical microscopy," J. Biomed. Opt. 14 (1), 1, (January/February) (2009).

[53] Clark, W. H., Jr., Anisworth,A. M., Bernardino,E. A., Yang,C., Mihm, M. C. and Reed, R. J., "The development biology of primary human malignant melanoma," Semin. Oncol. 2, (88-103) (1975). 